\title{
Calculating With Permanent Marker: How Blockchains Record Immutable Mistakes in Computational Chemistry
}

\author{
Magnus W. D. Hanson-Heine ${ }^{1 *}$ and Alexander P. Ashmore ${ }^{2}$ \\ ${ }^{I}$ School of Chemistry, University of Nottingham, University Park, Nottingham NG7 2RD, United \\ Kingdom. \\ ${ }^{2}$ School of Computing and Communications, The Open University, Walton Hall, Kents Hill, Milton \\ Keynes MK7 6AA, United Kingdom. \\ *magnus.hansonheine@nottingham.ac.uk
}




\section{Materials and Methods}

The output from the erroneous and corrected 10 step molecular dynamics simulations referenced in the main text are reported here, together with the corresponding source codes written in the Solidity programming language. The inputs and outputs of the "transactions" with the Ethereum blockchain that involved uploading the source code and running of the erroneous simulation have been analyzed using the website https://etherchain.org. The record of the simulation is available together with both sets of code on the Ethereum blockchain, and should remain available for review and replication independent of this publication for as long as the blockchain persists. The results in decimal form are also reported here in Table S1. The erroneous source code was uploaded to the Ethereum blockchain in block number 9359658 with the "contract address" 0x3d1a0b4e165c19a006562cfc14b44c4f83560249. Working knowledge of blockchain operations and how to use the Ethereum network, as well as a downloadable copy of the Ethereum blockchain itself can be found at the time of writing on the website https://ethereum.org/.

The mathematics used in these calculations makes use of decimal numbers. This is normally done in most programming languages through the use of doubles or floating point numbers, however, these are only precise up to a certain value. Two different computers generating slightly different results for the same double value can lead to slightly different simulation histories being recorded by different parts of the blockchain network. This can lead to the calculation being rejected as part of a consensus mechanism such as proof-of-work. Ethereum and similar blockchains that are capable of performing general computation have at the time of writing not yet implemented a method for calculating decimals with a fixed degree of precision, which would allow multiple machines to produce the same exact results from the same calculation. In this case, a third party library has been used in order to imitate floating point numbers using features that are currently available. In order to calculate these molecular dynamics trajectories, the ABDKMathQuad implements IEEE 754 standard fixed precision floating point numbers on the Ethereum blockchain. This library has been included in the code uploaded onto the blockchain as part of this work and can also be found at https://github.com/abdk-consulting/abdk-libraries-solidity/blob/master/ABDKMathQuad.md.

Using this library, the results have been scaled by $10^{\mathrm{p}}$ in order to provide accuracy up to $\mathrm{p}$ decimal places, where $\mathrm{p}$ is a variable supplied by the user when the code is called on the blockchain and chosen to be $\mathrm{p}=10$ for all of the calculations reported here. The output is then returned a set of integers by ABDKMathQuad and written as part of the simulation onto the blockchain as array of 256 bit integers in bytes displayed as a string of hexadecimal, and these have been divided by $10^{\mathrm{p}}$ before being reported as decimals in Table $\mathrm{S} 1$. 


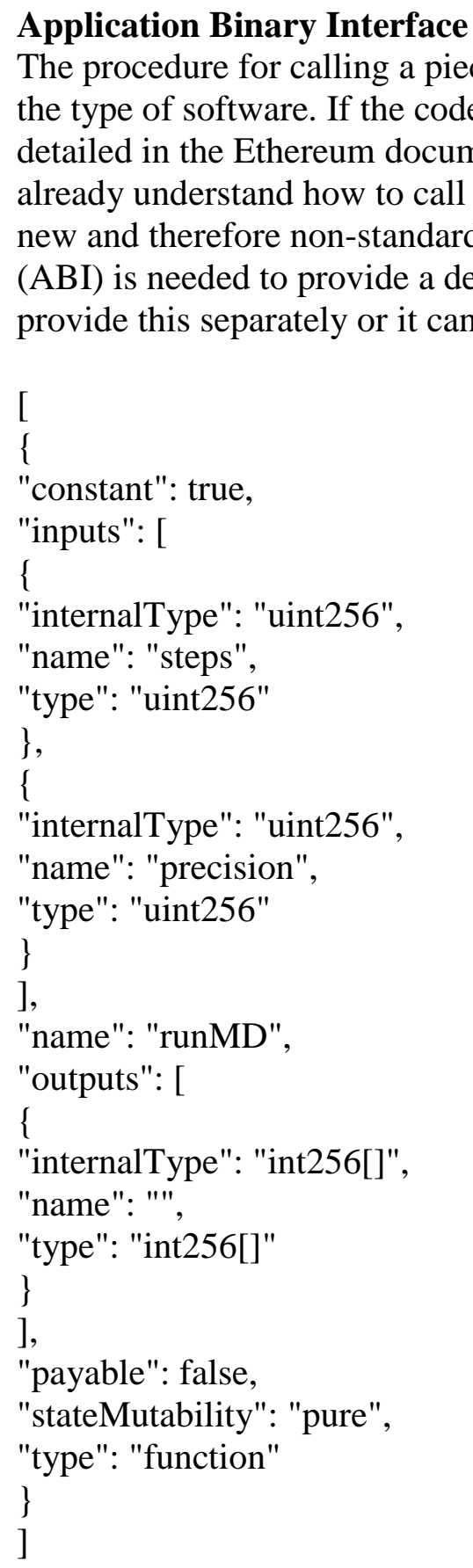
the type of software. If the code follows a standard format (such as the ERC20 token standard detailed in the Ethereum documentation) then most software for interacting with the code will already understand how to call it without the need for additional information. In the case of a new and therefore non-standard piece of software such as this, an Application Binary Interface (ABI) is needed to provide a description of the functions in the code. Developers will usually provide this separately or it can be generated from the source code. The ABI for this contract is: 


\section{Erroneous Molecular Dynamics Source Code}

The erroneous source code for the molecular dynamics simulation containing the mass error is given here:

pragma solidity ${ }^{\wedge} 0.5 .16$;

import "github.com/abdk-consulting/abdk-libraries-solidity/ABDKMathQuad.sol";

/***

* Copyright (c) 2019, ABDK Consulting

* All rights reserved.

* Redistribution and use in source and binary forms, with or without modification, are permitted provided that the following conditions are met:

* Redistributions of source code must retain the above copyright notice, this list of conditions and the following disclaimer.

* Redistributions in binary form must reproduce the above copyright notice, this list of conditions and the following disclaimer in the documentation and/or other materials provided with the distribution.

* All advertising materials mentioning features or use of this software must display the following acknowledgement: This product includes software developed by ABDK Consulting.

* Neither the name of ABDK Consulting nor the names of its contributors may be used to endorse or promote products derived from this software without specific prior written permission.

* THIS SOFTWARE IS PROVIDED BY ABDK CONSULTING "AS IS" AND ANY EXPRESS OR IMPLIED WARRANTIES, INCLUDING, BUT NOT LIMITED TO, THE IMPLIED WARRANTIES OF MERCHANTABILITY AND FITNESS FOR A PARTICULAR PURPOSE ARE DISCLAIMED. IN NO EVENT SHALL ABDK CONSULTING BE LIABLE FOR ANY DIRECT, INDIRECT, INCIDENTAL, SPECIAL, EXEMPLARY, OR CONSEQUENTIAL DAMAGES (INCLUDING, BUT NOT LIMITED TO, PROCUREMENT OF SUBSTITUTE GOODS OR SERVICES; LOSS OF USE, DATA, OR PROFITS; OR BUSINESS INTERRUPTION) HOWEVER CAUSED AND ON ANY THEORY OF LIABILITY, WHETHER IN CONTRACT, STRICT LIABILITY, OR TORT (INCLUDING NEGLIGENCE OR OTHERWISE) ARISING IN ANY WAY OUT OF THE USE OF THIS SOFTWARE, EVEN IF ADVISED OF THE POSSIBILITY OF SUCH DAMAGE. $* * /$

/// @ title Diatomic Molecular Dynamics

/// @ notice A diatomic molecular dynamics trajectory for the carbon monoxide molecule run with a variation of the velocity Verlet algorithm used to integrate Newton's equations of motion in atomic units.

III @ author Alex Ashmore <kezyma@yahoo.co.uk>, Magnus Hanson-Heine <magnus.hansonheine@gmail.com> contract DiatomicMD \{

bytes 16 constant $\operatorname{Re}=0 \mathrm{x} 400010 \mathrm{~d} 844 \mathrm{~d} 013 \mathrm{a} 92 \mathrm{a} 305532617 \mathrm{c} 1 \mathrm{bda}$; 
// 2.1316 - equilibrium bond length in a_0 (112.8 pm)

bytes 16 constant $\mathrm{K}=0 \mathrm{x} 3 \mathrm{fff} 310624 \mathrm{dd} 2 \mathrm{f} 1 \mathrm{a} 9 \mathrm{fbe} 76 \mathrm{c} 8 \mathrm{~b} 4395810$;

// 1.1915 - force constant in Eh/a_0^2 $(18.55 \mathrm{~N} / \mathrm{cm})$

bytes 16 constant $\mathrm{M} 1=0 \mathrm{x} 400 \mathrm{~d} 55 \mathrm{cc} 00000000000000000000000$;

// 21875 - mass of atom one in m_e (Carbon)

bytes 16 constant M2 $=0 x 400 \mathrm{dc} 793 \mathrm{c} 86594 \mathrm{af} 4 \mathrm{f0d} 844 \mathrm{~d} 013 \mathrm{a} 92 \mathrm{a} 3$;

// 29156.9457 - mass of atom two in m_e (Oxygen)

bytes 16 constant $\mathrm{dT}=0 \mathrm{x} 40010851 \mathrm{eb} 851 \mathrm{eb} 851 \mathrm{eb} 851 \mathrm{eb} 851 \mathrm{eb} 85$;

// $4.13 \quad-0.1$ fs time step in hbar/E_h

bytes 16 constant dTdTbyM1 2 = 0x3ff3986fded976d8bbb029473b7c7366;

$/ /((\mathrm{dT} * \mathrm{dT}) /(\mathrm{M} 1 * 2))$

bytes 16 constant dTdTbyM2x2 = 0x3ff332a2c7320b5680d44848021cdec4;

$/ /((\mathrm{dT} * \mathrm{dT}) /(\mathrm{M} 2 * 2))$

bytes 16 constant dTbyM1x2 $=0 \times 3 f f 18 b 94 a 3 c 45 c c a 7908 d 589 c b 4790 a 0$;

$/ /(\mathrm{dT} /(\mathrm{M} 1 * 2))$

bytes 16 constant dTbyM2x2 $=0 \times 3 f f 128 f b d f 4 c 5 c c d 459 b 646103 \mathrm{e} 7 \mathrm{e} 4 \mathrm{c} 6$;

$/ /(\mathrm{dT} /(\mathrm{M} 2 * 2))$

/// @ notice Initializes a molecular dynamics trajectory

I// @ param steps Number of steps in time in units of 0.1 femtosecond

I// @ param precision Number of decimal places to include in output

function runMD(uint256 steps, uint precision) public returns (int256[] memory) \{

bytes $16 \mathrm{r} 1=0 \mathrm{x} 400022430 \mathrm{e} 0729 \mathrm{bfecea} 7985 \mathrm{fddb} 6291$;

// 2.26767135 - initial bond length in a_0 $(120.0 \mathrm{pm})$

bytes $16 \mathrm{r} 2=0 \mathrm{x} 00000000000000000000000000000000 ; / / 0$

bytes $16 \mathrm{v} 1=0 \times 000000000000000000000000000000000 ; / / 0$

bytes $16 \mathrm{v} 2=0 \times 00000000000000000000000000000000 ; / / 0$

bytes $16 \mathrm{r}$;

bytes16 rMag;

bytes $16 \mathrm{f}$;

bytes 16 fNew;

uint $256 \mathrm{t}=0$;

uint ten $=10$;

uint multiplier $=$ ten $* *$ precision;

bytes 16 convertedMultiplier = ABDKMathQuad.fromUInt(multiplier);

int256[] memory results = new int256[](steps);

while $(\mathrm{t}<$ steps $)\{$

$\mathrm{r}=$ ABDKMathQuad.sub(r1,r2);

rMag = ABDKMathQuad.abs(r); 
f

ABDKMathQuad.div(ABDKMathQuad.mul(ABDKMathQuad.mul(ABDKMathQuad.neg(K),A BDKMathQuad.sub(rMag,Re)),r),rMag);

r1

ABDKMathQuad.add(ABDKMathQuad.add(r1,ABDKMathQuad.mul(dT,v1)),ABDKMathQuad .mul(dTdTbyM1x2,f));

$\mathrm{r} 2=$ ABDKMathQuad.sub(ABDKMathQuad.add(r2,ABDKMathQuad.mul(dT,v2)), ABDKMathQuad.mul(dTdTbyM2x2,f));

$\mathrm{r}=$ ABDKMathQuad.sub(r1,r2);

rMag = ABDKMathQuad.abs(r);

fNew

ABDKMathQuad.div(ABDKMathQuad.mul(ABDKMathQuad.mul(ABDKMathQuad.neg(K),A

BDKMathQuad.sub(rMag,Re)),r),rMag);

v1

ABDKMathQuad.add(v1,ABDKMathQuad.mul(dTbyM1x2,ABDKMathQuad.add(fNew,f))); v2

ABDKMathQuad.sub(v2,ABDKMathQuad.mul(dTbyM2x2,ABDKMathQuad.add(fNew,f)));

results $[\mathrm{t}]=\mathrm{ABDKMathQuad} \cdot \operatorname{toInt}(\mathrm{ABDKMathQuad}$.mul(rMag, convertedMultiplier));

$$
\mathrm{t}=\mathrm{t}+1
$$

\}

return results;

\}

\} 


\section{Erroneous Molecular Dynamics Source Code Upload Transaction Details}

The details of the transaction in which the erroneous source code containing the mass error was uploaded onto the Ethereum blockchain are presented here:

Block Number: 9359658

Hash: 0x79b1f485cf34fdca64f85b47f6ea36eb1b449a18b5a2f7de9e09cee86259adac

Time Stamp: 01/26/2020 8:26:25 PM Greenwich Mean Time (GMT)

From: 0xC120A82d25325c9700652D6f2288EBD2C4224567

To: 0x3d1A0B4e165C19A006562Cfc14b44C4F83560249

Fee: 0.00091 ETH (ca. \$0.16)

Gas Price: 0.0000000011 ETH

Gas Used: 825,139

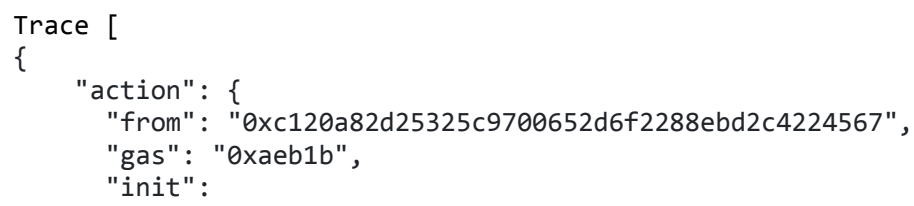

"0x608060405234801561001057600080fd5b50610df6806100206000396000f3fe608060405234801561001057600080fd5b50600 4361061002b5760003560e01c80639deac18d14610030575b600080fd5b6100536004803603604081101561004657600080fd5b508 $03590602001356100 a 3565 b 60408051602080825283518183015283519192839290830191858101910280838360005 b 83811015610$ 08f578181015183820152602001610077565b505050509050019250505060405180910390f35b60606f400022430e0729bfecea798 5fddb629160801b600080808080808080600a8b810a826100d1826102dd565b905060608f604051908082528060200260200182016 0405280156100ff578160200160208202803883390190505b5090505b8f8510156102c8576101158d8d610343565b9850610120896 1035c565b975061017561016f6101696101476f03fff310624dd2f1a9fbe76c8b43958160841b61036b565b6101648c6f2000086c2 26809d495182a9930be0ded60811b610343565b610374565b8b610374565b896105f2565b96506101bd6101a08e61019b6f4001085 1eb851eb851eb851eb851eb8560801b8f610374565b61087d565b61019b6f1ff9cc37ef6cbb6c5dd814a39dbe39b360811b8a61037 4565b9c506102056101e38d61019b6f40010851eb851eb851eb851eb851eb8560801b8e610374565b6102006f0ffccca8b1cc82d5a 0351212008737b160821b8a610374565b610343565b9b506102118d8d610343565b985061021c8961035c565b975061024361016f6 $101696101476 f 03 f f f 310624 d d 2 f 1 a 9 f b e 76 c 8 b 43958160841 b 61036 b 565 b 955061026 a 8 b 61019 b 6 f 01 f f 8 c 5 c a 51 e 22 e 653 c 846 a c 4$ e5a3c8560851b6101648a8c61087d565b9a506102918a6102006f1ff8947defa62e66a2cdb23081f3f26360811b6101648a8c61087 d565b99506102a56102a08984610374565b610c6b565b8186815181106102b157fe5b6020026020010181815250508460010194506 10103565b9c505050505050505050505050505b92915050565b6000816102ec5750600061033e565b8160006102f882610d1e565b9 $0506070811015610311578060700382901 b 9150610324565 b 6070811115610324576070810382901$ c91505b613fff0160701b60016 00160701b03919091161760801b90505b919050565b600061035583600160ff1b841861087d565b9392505050565b600160801b600 160ff1b031690565b600160ff1b1890565b6000617fff60f084811c8216919084901c8116908214156104245780617fff14156103f 2576001600160801b031985811690851614156103bf57505050600160ff1b811682186102d7565b600160ff1b6001600160801b031 98686181614156103e2575050508181176102d7565b5061ffff60ef1b91506102d79050565b600160801b600160ff1b03841661041 3575061ffff60ef1b91506102d79050565b505050600160ff1b811682186102d7565b80617fff141561046057600160801b600160f f1b03851661044f575061ffff60ef1b91506102d79050565b505050600160ff1b821681186102d7565b6001600160701b036080869 01c168261047c5760019250610483565b600160701b175b6001600160701b03608086901c168261049f57600192506104a6565b600 160701b175b90810290816104d457600160ff1b878718166104c35760006104c9565b600160ff1b5b9450505050506102d7565b928 $201926000600160 \mathrm{e} 11 \mathrm{~b} 83101561050857600160 \mathrm{e} 01 \mathrm{~b} 831015610500576104 \mathrm{fb} 83610 \mathrm{~d} 1 \mathrm{e} 565 \mathrm{~b} 610503565 \mathrm{~b} 60 \mathrm{e} 05 \mathrm{~b} 61050 \mathrm{~b} 565 \mathrm{~b} 60 \mathrm{e} 15$ b905061407081860110156105265760009450600092506105c3565b6140e081860110156105695761407085101561054b578461407 00383901c9250610560565b61407085111561056057614070850383901b92505b600094506105c3565b61c0dd81860111156105835 7617fff9450600092506105c3565b607081111561059a576070810383901c92506105ad565b60708110156105ad578060700383901 b92505b6001600160701b03831692506140df8186010394505b82607086901b888a186001607f1b60801b1660801c6001600160801 b0316171760801b955050505050506102d7565b6000617fff60f084811c8216919084901c8116908214156106275780617fff14156 10413575061 ffff60ef1b91506102d79050565b80617fff141561066b576dffffffffffffffffffffffffffffff60801b84161561065 a575061ffff60ef1b91506102d79050565b505050808218600160ff1b166102d7565b600160801b600160ff1b0384166106b557600 160801b600160ff1b03851661069d575061ffff60ef1b91506102d79050565b505050808218600160ff1b16617fff60f01b176102d 7565b6001600160701b03608085901c16816106d157600191506106d8565b600160701b175b6001600160701b03608087901c16836 $107175780156107125760006106 f c 82610 d 1$ e565b6001955060e20393840160711901939190911b90505b610721565b600160701b1 760721b5b81818161072a57fe5b0490508061074657600160ff1b878718166104c35760006104c9565b6001606c1b8110156107545 7fe5b6000600160731b82101561079457600160721b82101561078957600160711b821015610781576070610784565b60715b61078 c565b60725b60ff1661079d565b61079d82610d1e565b9050836140710181860111156107bb57617fff94506000915061084e565b8 $3818601613 \mathrm{ffc} 0110156107 \mathrm{~d} 657600094506000915061084 \mathrm{e} 565 \mathrm{~b} 83818601613 \mathrm{f} 8 \mathrm{c} 011015610823578385613 \mathrm{ffc} 011115610801578$ $385613 f f c 010382901 b 915061081 a 565 b 8385613 f f c 01101561081 a 57613 f f c 8585030382901$ c91505b6000945061084e565b60708 11115610836576070810382901 c91505b6001600160701b038216915083818601613f8d010394505b81607086901b888a186001607 f1b60801b1660801c6001600160801b0316171760801b955050505050506102d7565b6000617fff60f084811c8216919084901c811 6908214156108cb5780617fff14156108c1576001600160801b031985811690851614156103e25784925050506102d7565b8492505 0506102d7565b80617fff14156108df5783925050506102d7565b6001607f1b608086901c90811015906001600160701b031683610 
905576001935061090c565b600160701b175b6001607f1b608087901c90811015906001600160701b0316846109325760019450610 939565b600160701b175b82610969576001600160801b03198816600160ff1b14610959578761095c565b60005b965050505050505 06102d7565b80610989576001600160801b03198916600160ff1b14610959578861095c565b8486038415158315151415610aac576 0708113156109b057899750505050505050506102d7565b60008113156109cf57808260008212156109c657fe5b901c9150610a095 65b606f198112156109e857889750505050505050506102d7565b6000811215610a095780600003846000821215610a0157fe5b901 c93508596505b92810192600160711b8410610a24576001968701969390931c925b86617fff1415610a565784610a3e57617fff60f 01b610a48565b6001600160f01b03195b9750505050505050506102d7565b600160701b841015610a6b5760009650610a78565b600 1600160701b03841693505b83607088901b86610a8a576000610a90565b6001607f1b5b6001600160801b0316171760801b9750505 050505050506102d7565b6000811315610ac757600184901b9350600187039650610ade565b6000811215610ade57600182901b915 06001860396505b6070811315610af05760019150610b53565b6001811315610b185760018103600183036000821215610b0c57fe5 b901c6001019150610b53565b606f19811215610b2b5760019350610b53565b600019811215610b535760018160000303600185036 000821215610b4b57fe5b901c60010193505b818410610b64578184039350610b6d565b83820393508294505b83610b83575060009 6506102d795505050505050565b6000610b8e85610d1e565b90508060711415610bb457600185901c6001600160701b03169450600 $188019750610 c 03565 b 6070811015610 b f 657607081900380891115610 b e 3578086901 b 6001600160701 b 031695508089039850610$ bf0565b600098600019019590951b945b50610c03565b6001600160701b03851694505b87617fff1415610c365785610c1d57617ff f60f01b610c27565b6001600160f01b03195b985050505050505050506102d7565b84607089901b87610c48576000610c4e565b600 1607f1b5b6001600160801b0316171760801b985050505050505050506102d7565b6000617fff60f083901c166140fe 811115610c8 557600080fd5b613fff811015610c9957600091505061033e565b600160701b6001600160701b03608085901c161761406f8210156 10 cc35761406f8290031c610cd5565b61406f821115610cd55761406e1982011b5b6001607f1b608085901c10610d0157600160ff1 b811115610cf557600080fd5b600003915061033e9050565b6001600160ff1b03811115610d1557600080fd5b915061033e9050565 b6000808211610d2c57600080fd5b6000600160801b8310610d4157608092831c92015b680100000000000000008310610d5957604 092831c92015b6401000000008310610d6d57602092831c92015b620100008310610d7f57601092831c92015b6101008310610d905 7600892831c92015b60108310610da057600492831c92015b60048310610db057600292831c92015b600283106102d757600101929 1505056fea265627a7a72315820cfd2338ec7dec888beddf6f83e01e22812bc7cab40609b76a97ab7b44593e8cb64736f6c6343000 5100032",

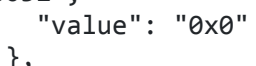

"blockHash": "0x49bfcd06c26a20427b84ce98efcbd084adc1ec9fcc5dba5ccf2476495f8048ba",

"blockNumber": 9359658,

"result": \{

"address": "0x3d1a0b4e165c19a006562cfc14b44c4f83560249",

"code" :

"0x608060405234801561001057600080fd5b506004361061002b5760003560e01c80639deac18d14610030575b600080fd5b61005 $36004803603604081101561004657600080 f d 5 b 50803590602001356100 a 3565 b 60408051602080825283518183015283519192839$ 290830191858101910280838360005 b8381101561008f578181015183820152602001610077565b505050509050019250505060405 180910390f35b60606f400022430e0729bfecea7985fddb629160801b600080808080808080600a8b810a826100d1826102dd565b9 $05060608 f 6040519080825280602002602001820160405280156100 f f 578160200160208202803883390190505 b 5090505 b 8 f 85101$ 56102c8576101158d8d610343565b98506101208961035c565b975061017561016f6101696101476f03fff310624dd2f1a9fbe76c8 b43958160841b61036b565b6101648c6f2000086c226809d495182a9930be0ded60811b610343565b610374565b8b610374565b896 105f2565b96506101bd6101a08e61019b6f40010851eb851eb851eb851eb851eb8560801b8f610374565b61087d565b61019b6f1ff 9cc37ef6cbb6c5dd814a39dbe39b360811b8a610374565b9c506102056101e38d61019b6f40010851eb851eb851eb851eb851eb856 0801b8e610374565b6102006f0ffccca8b1cc82d5a0351212008737b160821b8a610374565b610343565b9b506102118d8d6103435 65b985061021c8961035c565b975061024361016f6101696101476f03fff310624dd2f1a9fbe76c8b43958160841b61036b565b955 061026a8b61019b6f01ff8c5ca51e22e653c846ac4e5a3c8560851b6101648a8c61087d565b9a506102918a6102006f1ff8947defa 62e66a2cdb23081f3f26360811b6101648a8c61087d565b99506102a56102a08984610374565b610c6b565b8186815181106102b15 7fe5b602002602001018181525050846001019450610103565b9c505050505050505050505050505b92915050565b6000816102ec5 750600061033 e565b8160006102f882610d1e565b90506070811015610311578060700382901b9150610324565b607081111561032 4576070810382901c91505b613fff0160701b6001600160701b03919091161760801b90505b919050565b600061035583600160ff1 b841861087d565b9392505050565b600160801b600160ff1b031690565b600160ff1b1890565b6000617fff60f084811c821691908 4901c8116908214156104245780617fff14156103f2576001600160801b031985811690851614156103bf57505050600160ff1b811 682186102d7565b600160ff1b6001600160801b03198686181614156103e2575050508181176102d7565b5061ffff60ef1b9150610 2d79050565b600160801b600160ff1b038416610413575061ffff60ef1b91506102d79050565b505050600160ff1b811682186102d 7565b80617fff141561046057600160801b600160ff1b03851661044f575061ffff60ef1b91506102d79050565b505050600160ff1 b821681186102d7565b6001600160701b03608086901c168261047c5760019250610483565b600160701b175b6001600160701b036 08086901c168261049f57600192506104a6565b600160701b175b90810290816104d457600160ff1b878718166104c35760006104c 9565b600160ff1b5b9450505050506102d7565b928201926000600160e11b83101561050857600160e01b831015610500576104fb8 3610d1e565b610503565b60e05b61050b565b60e15b905061407081860110156105265760009450600092506105c3565b6140e0818 60110156105695761407085101561054b57846140700383901c9250610560565b61407085111561056057614070850383901b92505 b600094506105c3565b61c0dd818601111561058357617fff9450600092506105c3565b607081111561059a576070810383901c925 06105ad565b60708110156105ad578060700383901b92505b6001600160701b03831692506140df8186010394505b82607086901b8 88a186001607f1b60801b1660801c6001600160801b0316171760801b955050505050506102d7565b6000617fff60f084811c82169 19084901c8116908214156106275780617fff1415610413575061ffff60ef1b91506102d79050565b80617fff141561066b576dfff fffffffffffffffffffffffff60801b84161561065a575061ffff60ef1b91506102d79050565b505050808218600160ff1b166102d 7565b600160801b600160ff1b0384166106b557600160801b600160ff1b03851661069d575061ffff60ef1b91506102d79050565b5 05050808218600160ff1b16617fff60f01b176102d7565b6001600160701b03608085901c16816106d157600191506106d8565b600 160701b175b6001600160701b03608087901c16836107175780156107125760006106fc82610d1e565b6001955060e203938401607 11901939190911b90505b610721565b600160701b1760721b5b81818161072a57fe5b0490508061074657600160ff1b87871816610 4c35760006104c9565b6001606c1b81101561075457fe5b6000600160731b82101561079457600160721b821015610789576001607 11b821015610781576070610784565b60715b61078c565b60725b60ff1661079d565b61079d82610d1e565b9050836140710181860 
111156107bb57617fff94506000915061084e565b83818601613ffc0110156107d657600094506000915061084e565b83818601613 f8c011015610823578385613ffc011115610801578385613ffc010382901b915061081a565b8385613ffc01101561081a57613ffc8 585030382901c91505b6000945061084e565b6070811115610836576070810382901c91505b6001600160701b03821691508381860 1613f8d010394505b81607086901b888a186001607f1b60801b1660801c6001600160801b0316171760801b955050505050506102d 7565b6000617fff60f084811c8216919084901c8116908214156108cb5780617ffff14156108c1576001600160801b0319858116908 51614156103e25784925050506102d7565b84925050506102d7565b80617fff14156108df5783925050506102d7565b6001607f1b6 08086901c90811015906001600160701b031683610905576001935061090c565b600160701b175b6001607f1b608087901c9081101 5906001600160701b0316846109325760019450610939565b600160701b175b82610969576001600160801b03198816600160ff1b1 4610959578761095c565b60005b96505050505050506102d7565b80610989576001600160801b03198916600160ff1b14610959578 861095c565b8486038415158315151415610aac5760708113156109b057899750505050505050506102d7565b60008113156109cf5 7808260008212156109c657fe5b901c9150610a09565b606f198112156109e857889750505050505050506102d7565b60008112156 10a095780600003846000821215610a0157fe5b901c93508596505b92810192600160711b8410610a24576001968701969390931c9 25b86617fff1415610a565784610a3e57617fff60f01b610a48565b6001600160f01b03195b9750505050505050506102d7565b600 160701b841015610a6b5760009650610a78565b6001600160701b03841693505b83607088901b86610a8a576000610a90565b60016 07f1b5b6001600160801b0316171760801b9750505050505050506102d7565b6000811315610ac757600184901b935060018703965 0610ade565b6000811215610ade57600182901b91506001860396505b6070811315610af05760019150610b53565b6001811315610 b185760018103600183036000821215610b0c57fe5b901c6001019150610b53565b606f19811215610b2b5760019350610b53565b6 $00019811215610 b 535760018160000303600185036000821215610 b 4 b 57 f e 5 b 901 c 60010193505 b 818410610 b 64578184039350610$ b6d565b83820393508294505b83610b835750600096506102d795505050505050565b6000610b8e85610d1e565b905080607114156 10bb457600185901c6001600160701b03169450600188019750610c03565b6070811015610bf657607081900380891115610be3578 086901b6001600160701b031695508089039850610bf0565b600098600019019590951b945b50610c03565b6001600160701b03851 694505b87617fff1415610c365785610c1d57617fff60f01b610c27565b6001600160f01b03195b985050505050505050506102d75 65b84607089901b87610c48576000610c4e565b6001607f1b5b6001600160801b0316171760801b985050505050505050506102d 75 65b6000617fff60f083901c166140fe811115610c8557600080fd5b613fff811015610c9957600091505061033e565b600160701b6 $001600160701 \mathrm{~b} 03608085901 \mathrm{c} 161761406 \mathrm{f} 821015610 \mathrm{cc} 35761406 \mathrm{f} 8290031 \mathrm{c} 610 \mathrm{~cd} 5565 \mathrm{~b} 61406 \mathrm{f} 821115610 \mathrm{~cd} 55761406 \mathrm{e} 1982011$ b5b6001607f1b608085901c10610d0157600160ff1b811115610cf557600080fd5b600003915061033e9050565b6001600160ff1b0 $3811115610 d 1557600080 f d 5 b 915061033 e 9050565 b 6000808211610 d 2 c 57600080 f d 5 b 6000600160801 b 8310610 d 4157608092831$ c92015b680100000000000000008310610d5957604092831c92015b6401000000008310610d6d57602092831c92015b62010000831 0610d7f57601092831c92015b6101008310610d9057600892831c92015b60108310610da057600492831c92015b60048310610db05 7600292831c92015b600283106102d7576001019291505056fea265627a7a72315820cfd2338ec7dec888beddf6f83e01e22812bc7 cab40609b76a97ab7b44593e8cb64736f6c63430005100032", \} , "gasUsed": "0xaeb1b"

"subtraces": 0 ,

"traceAddress": [],

"transactionHash": "0x79b1f485cf34fdca64f85b47f6ea36eb1b449a18b5a2f7de9e09cee86259adac",

"transactionPosition": 77,

"type": "create" \}

] 


\section{Erroneous Molecular Dynamics Trajectory Transaction Details}

The details of the transaction and the corresponding blockchain output from the erroneous $1 \mathrm{fs}$ molecular dynamics preliminary simulation on the Ethereum blockchain are presented here:

Block Number: 9359692

Hash: 0x59d887b7909685b2acb35e2757f5ccacbf6097a393ab286b347d930988500e73

Time Stamp: 01/26/2020 08:34:42 PM Greenwich Mean Time (GMT)

From: 0xC120A82d25325c9700652D6f2288EBD2C4224567

To: 0x3d1A0B4e165C19A006562Cfc14b44C4F83560249

Fee: 0.00026 ETH (ca. \$0.07)

Gas Price: 0.0000000011 ETH

Gas Used: 238,619

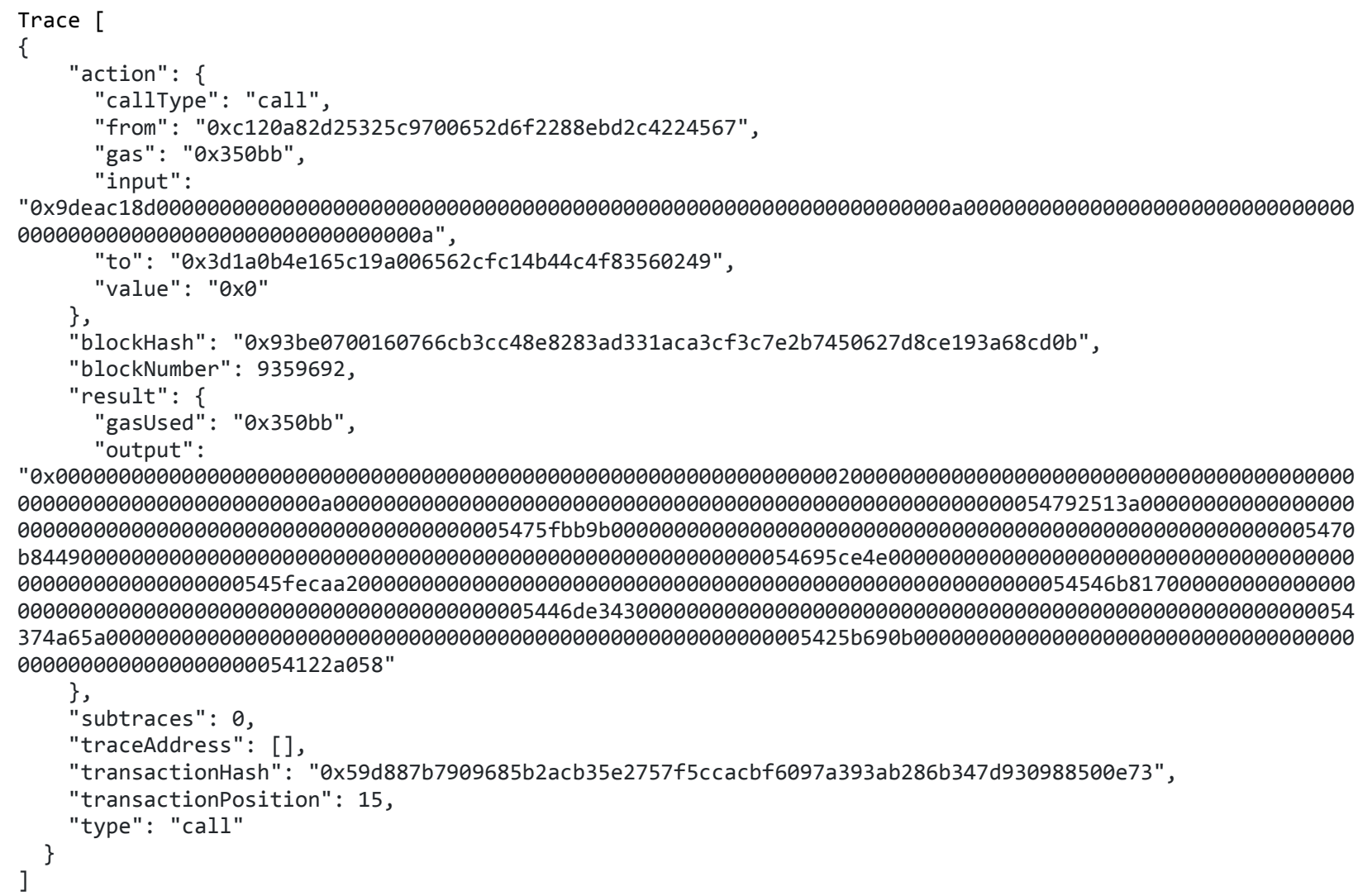

"0x0000000000000000000000000000000000000000000000000000000000000020000000000000000000000000000000000000000 $000000000000000000000000 \mathrm{a} 000000000000000000000000000000000000000000000000000000054792513 a 00000000000000000$ $000000000000000000000000000000000000005475 \mathrm{fbb} 9 \mathrm{~b} 00000000000000000000000000000000000000000000000000000005470$ b8449000000000000000000000000000000000000000000000000000000054695ce4e0000000000000000000000000000000000000

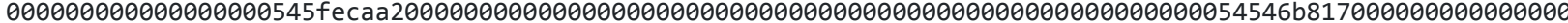
$00000000000000000000000000000000000000005446 \mathrm{de} 343000000000000000000000000000000000000000000000000000000054$ 374a65a00000000000000000000000000000000000000000000000000000005425b690b00000000000000000000000000000000000 $0000000000000000000054122 \mathrm{a} 058 "$ 


\section{Corrected Molecular Dynamics Source Code}

The source code for the molecular dynamics simulation that was used to run the corrected simulations on the Ethereum blockchain is given here:

pragma solidity ${ }^{\wedge} 0.5 .16$;

import "github.com/abdk-consulting/abdk-libraries-solidity/ABDKMathQuad.sol";

/***

* Copyright (c) 2019, ABDK Consulting

* All rights reserved.

* Redistribution and use in source and binary forms, with or without modification, are permitted provided that the following conditions are met:

* Redistributions of source code must retain the above copyright notice, this list of conditions and the following disclaimer.

* Redistributions in binary form must reproduce the above copyright notice, this list of conditions and the following disclaimer in the documentation and/or other materials provided with the distribution.

* All advertising materials mentioning features or use of this software must display the following acknowledgement: This product includes software developed by ABDK Consulting.

* Neither the name of ABDK Consulting nor the names of its contributors may be used to endorse or promote products derived from this software without specific prior written permission.

* THIS SOFTWARE IS PROVIDED BY ABDK CONSULTING "AS IS" AND ANY EXPRESS OR IMPLIED WARRANTIES, INCLUDING, BUT NOT LIMITED TO, THE IMPLIED WARRANTIES OF MERCHANTABILITY AND FITNESS FOR A PARTICULAR PURPOSE ARE DISCLAIMED. IN NO EVENT SHALL ABDK CONSULTING BE LIABLE FOR ANY DIRECT, INDIRECT, INCIDENTAL, SPECIAL, EXEMPLARY, OR CONSEQUENTIAL DAMAGES (INCLUDING, BUT NOT LIMITED TO, PROCUREMENT OF SUBSTITUTE GOODS OR SERVICES; LOSS OF USE, DATA, OR PROFITS; OR BUSINESS INTERRUPTION) HOWEVER CAUSED AND ON ANY THEORY OF LIABILITY, WHETHER IN CONTRACT, STRICT LIABILITY, OR TORT (INCLUDING NEGLIGENCE OR OTHERWISE) ARISING IN ANY WAY OUT OF THE USE OF THIS SOFTWARE, EVEN IF ADVISED OF THE POSSIBILITY OF SUCH DAMAGE. $* * /$

/// @ title Diatomic Molecular Dynamics

/// @ notice A diatomic molecular dynamics trajectory for the carbon monoxide molecule run with a variation of the velocity Verlet algorithm used to integrate Newton's equations of motion in atomic units.

III @ author Alex Ashmore <kezyma@yahoo.co.uk>, Magnus Hanson-Heine <magnus.hansonheine@gmail.com> contract DiatomicMD \{

bytes 16 constant $\mathrm{Re}=0 \mathrm{x} 400010 \mathrm{~d} 844 \mathrm{~d} 013 \mathrm{a} 92 \mathrm{a} 305532617 \mathrm{c} 1 \mathrm{bda}$; 
// 2.1316 - equilibrium bond length in a_0 (112.8 pm)

bytes 16 constant $\mathrm{K}=0 \mathrm{x} 3 \mathrm{fff} 310624 \mathrm{dd} 2 \mathrm{f} 1 \mathrm{a} 9 \mathrm{fbe} 76 \mathrm{c} 8 \mathrm{~b} 4395810$;

// 1.1915 - force constant in Eh/a_0^2 $(18.55 \mathrm{~N} / \mathrm{cm})$

bytes 16 constant $\mathrm{M} 1=0 \mathrm{x} 400 \mathrm{~d} 55 \mathrm{cc} 00000000000000000000000$;

// 21875 - mass of atom one in m_e (Carbon)

bytes 16 constant M2 $=0 x 400 \mathrm{dc} 793 \mathrm{c} 86594 \mathrm{af} 4 \mathrm{f0d} 844 \mathrm{~d} 013 \mathrm{a} 92 \mathrm{a} 3$;

// 29156.9457 - mass of atom two in m_e (Oxygen)

bytes 16 constant $\mathrm{dT}=0 \mathrm{x} 40010851 \mathrm{eb} 851 \mathrm{eb} 851 \mathrm{eb} 851 \mathrm{eb} 851 \mathrm{eb} 85$;

// $4.13-0.1$ fs time step in hbar/E_h

bytes 16 constant dTdTbyM1x2 = 0x3ff398cf77d58230a4ec10bd70161b26;

$/ /((\mathrm{dT} * \mathrm{dT}) /(\mathrm{M} 1 * 2))$

bytes 16 constant dTdTbyM2x2 = 0x3ff332b5c5317be96318c8a728b2194e;

$/ /((\mathrm{dT} * \mathrm{dT}) /(\mathrm{M} 2 * 2))$

bytes 16 constant dTbyM1x2 = 0x3ff18bf13a6a5f196b0b10d53a599821;

// $(\mathrm{dT} /(\mathrm{M} 1 * 2))$

bytes 16 constant dTbyM2x2 = 0x3ff1290e444148485119dcfda2c40c1e;

$/ /(\mathrm{dT} /(\mathrm{M} 2 * 2))$

/// @ notice Initializes a molecular dynamics trajectory

/I/ @ param steps Number of steps in time in units of 0.1 femtosecond

/// @ param precision Number of decimal places to include in output

function runMD(uint256 steps, uint precision) public returns (int256[] memory) \{

bytes16 $\mathrm{r} 1=0 \mathrm{x} 400022430 \mathrm{e} 0729 \mathrm{bfecea} 7985 f d d b 6291$; / 2.26767135 - initial bond length in a_0 $(120.0 \mathrm{pm})$

bytes $16 \mathrm{r} 2=0 \mathrm{x} 00000000000000000000000000000000 ; / / 0$

bytes $16 \mathrm{v} 1=0 \times 00000000000000000000000000000000 ; / / 0$

bytes $16 \mathrm{v} 2=0 \times 00000000000000000000000000000000 ; / / 0$

bytes $16 \mathrm{r}$;

bytes16 rMag;

bytes $16 \mathrm{f}$;

bytes 16 fNew;

uint $256 \mathrm{t}=0$;

uint ten $=10$;

uint multiplier $=$ ten $* *$ precision;

bytes 16 convertedMultiplier = ABDKMathQuad.fromUInt(multiplier);

int256[] memory results $=$ new int256[](steps);

while $(\mathrm{t}<$ steps $)\{$

$\mathrm{r}=\mathrm{ABDKMathQuad} . \mathrm{sub}(\mathrm{r} 1, \mathrm{r} 2)$;

rMag = ABDKMathQuad.abs(r); 
f

ABDKMathQuad.div(ABDKMathQuad.mul(ABDKMathQuad.mul(ABDKMathQuad.neg(K),A BDKMathQuad.sub(rMag,Re)),r),rMag);

r1

ABDKMathQuad.add(ABDKMathQuad.add(r1,ABDKMathQuad.mul(dT,v1)),ABDKMathQuad .mul(dTdTbyM1x2,f));

$\mathrm{r} 2=$ ABDKMathQuad.sub(ABDKMathQuad.add(r2,ABDKMathQuad.mul(dT,v2)), ABDKMathQuad.mul(dTdTbyM2x2,f));

$\mathrm{r}=$ ABDKMathQuad.sub(r1,r2);

rMag = ABDKMathQuad.abs(r);

fNew

ABDKMathQuad.div(ABDKMathQuad.mul(ABDKMathQuad.mul(ABDKMathQuad.neg(K),A

BDKMathQuad.sub(rMag,Re)),r),rMag);

v1

ABDKMathQuad.add(v1,ABDKMathQuad.mul(dTbyM1x2,ABDKMathQuad.add(fNew,f))); v2

ABDKMathQuad.sub(v2,ABDKMathQuad.mul(dTbyM2x2,ABDKMathQuad.add(fNew,f)));

results $[\mathrm{t}]=\mathrm{ABDKMathQuad} \cdot \operatorname{toInt}(\mathrm{ABDKMathQuad}$.mul(rMag, convertedMultiplier)); $\mathrm{t}=\mathrm{t}+1$

\}

return results;

\} 


\section{Corrected Molecular Dynamics Source Code Upload Transaction Details}

The details of the transaction in which the source code for the molecular dynamics simulation was uploaded onto the Ethereum blockchain are presented here:

Block Number: 9360156

Hash: 0x1c98dbb671dbe76b7cb4188d7585296e5adbe0f64fe8144546b4a82081089152

Time Stamp: 01/26/2020 10:25:44 PM Greenwich Mean Time (GMT)

From: 0xC120A82d25325c9700652D6f2288EBD2C4224567

To: 0x9680E167F6221ea0A9dcE066A4f9c769621bC577

Fee: 0.00091 ETH (ca. \$0.15)

Gas Price: 0.0000000011 ETH

Gas Used: 825,151

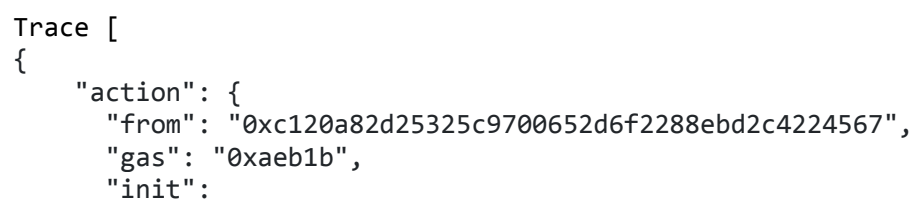

"0x608060405234801561001057600080fd5b50610df6806100206000396000f3fe608060405234801561001057600080fd5b50600 4361061002b5760003560e01c80639deac18d14610030575b600080fd5b6100536004803603604081101561004657600080fd5b508 $03590602001356100 a 3565 b 60408051602080825283518183015283519192839290830191858101910280838360005 b 83811015610$ 08f578181015183820152602001610077565b505050509050019250505060405180910390f35b60606f400022430e0729bfecea798 5fddb629160801b600080808080808080600a8b810a826100d1826102dd565b905060608f604051908082528060200260200182016 0405280156100ff578160200160208202803883390190505b5090505b8f8510156102c8576101158d8d610343565b9850610120896 1035c565b975061017561016f6101696101476f03fff310624dd2f1a9fbe76c8b43958160841b61036b565b6101648c6f2000086c2 26809d495182a9930be0ded60811b610343565b610374565b8b610374565b896105f2565b96506101bd6101a08e61019b6f4001085 1eb851eb851eb851eb851eb8560801b8f610374565b61087d565b61019b6f1ff9cc67bbeac1185276085eb80b0d9360811b8a61037 4565b9c506102056101e38d61019b6f40010851eb851eb851eb851eb851eb8560801b8e610374565b6102006f1ff9995ae298bdf4b 18c645394590ca760811b8a610374565b610343565b9b506102118d8d610343565b985061021c8961035c565b975061024361016f6 101696101476f03fff310624dd2f1a9fbe76c8b43958160841b61036b565b955061026a8b61019b6f3ff18bf13a6a5f196b0b10d53 a59982160801b6101648a8c61087d565b9a506102918a6102006f1ff894872220a424288cee7ed162060f60811b6101648a8c61087 d565b99506102a56102a08984610374565b610c6b565b8186815181106102b157fe5b6020026020010181815250508460010194506 10103565b9c505050505050505050505050505b92915050565b6000816102ec5750600061033e565b8160006102f882610d1e565b9 $0506070811015610311578060700382901 b 9150610324565 b 6070811115610324576070810382901$ c91505b613fff0160701b60016 00160701b03919091161760801b90505b919050565b600061035583600160ff1b841861087d565b9392505050565b600160801b600 160ff1b031690565b600160ff1b1890565b6000617fff60f084811c8216919084901c8116908214156104245780617fff14156103f 2576001600160801b031985811690851614156103bf57505050600160ff1b811682186102d7565b600160ff1b6001600160801b031 98686181614156103e2575050508181176102d7565b5061ffff60ef1b91506102d79050565b600160801b600160ff1b03841661041 3575061ffff60ef1b91506102d79050565b505050600160ff1b811682186102d7565b80617fff141561046057600160801b600160f f1b03851661044f575061ffff60ef1b91506102d79050565b505050600160ff1b821681186102d7565b6001600160701b036080869 01c168261047c5760019250610483565b600160701b175b6001600160701b03608086901c168261049f57600192506104a6565b600 160701b175b90810290816104d457600160ff1b878718166104c35760006104c9565b600160ff1b5b9450505050506102d7565b928 201926000600160e11b83101561050857600160e01b831015610500576104fb83610d1e565b610503565b60e05b61050b565b60e15 b905061407081860110156105265760009450600092506105c3565b6140e081860110156105695761407085101561054b578461407 00383901c9250610560565b61407085111561056057614070850383901b92505b600094506105c3565b61c0dd81860111156105835 7617fff9450600092506105c3565b607081111561059a576070810383901c92506105ad565b60708110156105ad578060700383901 b92505b6001600160701b03831692506140df8186010394505b82607086901b888a186001607f1b60801b1660801c6001600160801 b0316171760801b955050505050506102d7565b6000617fff60f084811c8216919084901c8116908214156106275780617fff14156 10413575061 ffff60ef1b91506102d79050565b80617fff141561066b576dffffffffffffffffffffffffffffff60801b84161561065 a575061ffff60ef1b91506102d79050565b505050808218600160ff1b166102d7565b600160801b600160ff1b0384166106b557600 160801b600160ff1b03851661069d575061ffff60ef1b91506102d79050565b505050808218600160ff1b16617fff60f01b176102d 7565b6001600160701b03608085901c16816106d157600191506106d8565b600160701b175b6001600160701b03608087901c16836 $107175780156107125760006106 f c 82610 d 1$ e565b6001955060e20393840160711901939190911b90505b610721565b600160701b1 760721b5b81818161072a57fe5b0490508061074657600160ff1b878718166104c35760006104c9565b6001606c1b8110156107545 7fe5b6000600160731b82101561079457600160721b82101561078957600160711b821015610781576070610784565b60715b61078 c565b60725b60ff1661079d565b61079d82610d1e565b9050836140710181860111156107bb57617fff94506000915061084e565b8 $3818601613 \mathrm{ffc} 0110156107 \mathrm{~d} 657600094506000915061084 \mathrm{e} 565 \mathrm{~b} 83818601613 \mathrm{f} 8 \mathrm{c} 011015610823578385613 \mathrm{ffc} 011115610801578$ $385613 f f c 010382901 b 915061081 a 565 b 8385613 f f c 01101561081 a 57613 f f c 8585030382901$ c91505b6000945061084e565b60708 11115610836576070810382901 c91505b6001600160701b038216915083818601613f8d010394505b81607086901b888a186001607 f1b60801b1660801c6001600160801b0316171760801b955050505050506102d7565b6000617fff60f084811c8216919084901c811 6908214156108cb5780617fff14156108c1576001600160801b031985811690851614156103e25784925050506102d7565b8492505 0506102d7565b80617fff14156108df5783925050506102d7565b6001607f1b608086901c90811015906001600160701b031683610 
905576001935061090c565b600160701b175b6001607f1b608087901c90811015906001600160701b0316846109325760019450610 939565b600160701b175b82610969576001600160801b03198816600160ff1b14610959578761095c565b60005b965050505050505 06102d7565b80610989576001600160801b03198916600160ff1b14610959578861095c565b8486038415158315151415610aac576 0708113156109b057899750505050505050506102d7565b60008113156109cf57808260008212156109c657fe5b901c9150610a095 65b606f198112156109e857889750505050505050506102d7565b6000811215610a095780600003846000821215610a0157fe5b901 c93508596505b92810192600160711b8410610a24576001968701969390931c925b86617fff1415610a565784610a3e57617fff60f 01b610a48565b6001600160f01b03195b9750505050505050506102d7565b600160701b841015610a6b5760009650610a78565b600 1600160701b03841693505b83607088901b86610a8a576000610a90565b6001607f1b5b6001600160801b0316171760801b9750505 050505050506102d7565b6000811315610ac757600184901b9350600187039650610ade565b6000811215610ade57600182901b915 06001860396505b6070811315610af05760019150610b53565b6001811315610b185760018103600183036000821215610b0c57fe5 b901c6001019150610b53565b606f19811215610b2b5760019350610b53565b600019811215610b535760018160000303600185036 000821215610b4b57fe5b901c60010193505b818410610b64578184039350610b6d565b83820393508294505b83610b83575060009 6506102d795505050505050565b6000610b8e85610d1e565b90508060711415610bb457600185901c6001600160701b03169450600 $188019750610 c 03565 b 6070811015610 b f 657607081900380891115610 b e 3578086901 b 6001600160701 b 031695508089039850610$ bf0565b600098600019019590951b945b50610c03565b6001600160701b03851694505b87617fff1415610c365785610c1d57617ff f60f01b610c27565b6001600160f01b03195b985050505050505050506102d7565b84607089901b87610c48576000610c4e565b600 $1607 f 1 b 5 b 6001600160801 b 0316171760801 b 985050505050505050506102 d 7565 b 6000617 f f f 60 f 083901 c 166140 f e 811115610 c 8$ 557600080fd5b613fff811015610c9957600091505061033e565b600160701b6001600160701b03608085901c161761406f8210156 10 cc35761406f8290031c610cd5565b61406f821115610cd55761406e1982011b5b6001607f1b608085901c10610d0157600160ff1 b811115610cf557600080fd5b600003915061033e9050565b6001600160ff1b03811115610d1557600080fd5b915061033e9050565 b6000808211610d2c57600080fd5b6000600160801b8310610d4157608092831c92015b680100000000000000008310610d5957604 092831c92015b6401000000008310610d6d57602092831c92015b620100008310610d7f57601092831c92015b6101008310610d905 7600892831 c92015b60108310610da057600492831c92015b60048310610db057600292831c92015b600283106102d757600101929 1505056fea265627a7a72315820d72739475f1b26be10735eab39ccaf43992cf00f66b7e7fbdb4a3cad038216a264736f6c6343000 5100032",

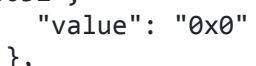

"blockHash": "0x82e933a530bf6299df1522de1c4a2cca609d2d2ec13cd04a07fced2b2062bb78",

"blockNumber": 9360156 ,

"result": \{

"address": "0x9680e167f6221ea0a9dce066a4f9c769621bc577",

"code" :

"0x608060405234801561001057600080fd5b506004361061002b5760003560e01c80639deac18d14610030575b600080fd5b61005 $36004803603604081101561004657600080 f d 5 b 50803590602001356100 a 3565 b 60408051602080825283518183015283519192839$ $290830191858101910280838360005 b 8381101561008 f 578181015183820152602001610077565 b 505050509050019250505060405$ 180910390f35b60606f400022430e0729bfecea7985fddb629160801b600080808080808080600a8b810a826100d1826102dd565b9 $05060608 f 6040519080825280602002602001820160405280156100 f f 578160200160208202803883390190505 b 5090505 b 8 f 85101$ 56102c8576101158d8d610343565b98506101208961035c565b975061017561016f6101696101476f03fff310624dd2f1a9fbe76c8 b43958160841b61036b565b6101648c6f2000086c226809d495182a9930be0ded60811b610343565b610374565b8b610374565b896 105f2565b96506101bd6101a08e61019b6f40010851eb851eb851eb851eb851eb8560801b8f610374565b61087d565b61019b6f1ff 9cc67bbeac1185276085eb80b0d9360811b8a610374565b9c506102056101e38d61019b6f40010851eb851eb851eb851eb851eb856 0801b8e610374565b6102006f1ff9995ae298bdf4b18c645394590ca760811b8a610374565b610343565b9b506102118d8d6103435 65b985061021c8961035c565b975061024361016f6101696101476f03fff310624dd2f1a9fbe76c8b43958160841b61036b565b955 061026a8b61019b6f3ff18bf13a6a5f196b0b10d53a59982160801b6101648a8c61087d565b9a506102918a6102006f1ff89487222 0a424288cee7ed162060f60811b6101648a8c61087d565b99506102a56102a08984610374565b610c6b565b8186815181106102b15 7fe5b602002602001018181525050846001019450610103565b9c505050505050505050505050505b92915050565b6000816102ec5 750600061033 e565b8160006102f882610d1e565b90506070811015610311578060700382901b9150610324565b607081111561032 4576070810382901c91505b613fff0160701b6001600160701b03919091161760801b90505b919050565b600061035583600160ff1 b841861087d565b9392505050565b600160801b600160ff1b031690565b600160ff1b1890565b6000617fff60f084811c821691908 4901c8116908214156104245780617fff14156103f2576001600160801b031985811690851614156103bf57505050600160ff1b811 682186102d7565b600160ff1b6001600160801b03198686181614156103e2575050508181176102d7565b5061ffff60ef1b9150610 2d79050565b600160801b600160ff1b038416610413575061ffff60ef1b91506102d79050565b505050600160ff1b811682186102d 7565 b80617fff141561046057600160801b600160ff1b03851661044f575061ffff60ef1b91506102d79050565b505050600160ff1 b821681186102d7565b6001600160701b03608086901c168261047c5760019250610483565b600160701b175b6001600160701b036 08086901c168261049f57600192506104a6565b600160701b175b90810290816104d457600160ff1b878718166104c35760006104c 9565b600160ff1b5b9450505050506102d7565b928201926000600160e11b83101561050857600160e01b831015610500576104fb8 3610d1e565b610503565b60e05b61050b565b60e15b905061407081860110156105265760009450600092506105c3565b6140e0818 60110156105695761407085101561054b57846140700383901c9250610560565b61407085111561056057614070850383901b92505 b600094506105c3565b61c0dd818601111561058357617fff9450600092506105c3565b607081111561059a576070810383901c925 06105ad565b60708110156105ad578060700383901b92505b6001600160701b03831692506140df8186010394505b82607086901b8 88a186001607f1b60801b1660801c6001600160801b0316171760801b955050505050506102d7565b6000617fff60f084811c82169 19084901c8116908214156106275780617fff1415610413575061ffff60ef1b91506102d79050565b80617fff141561066b576dfff fffffffffffffffffffffffff60801b84161561065a575061ffff60ef1b91506102d79050565b505050808218600160ff1b166102d 7565b600160801b600160ff1b0384166106b557600160801b600160ff1b03851661069d575061ffff60ef1b91506102d79050565b5 05050808218600160ff1b16617fff60f01b176102d7565b6001600160701b03608085901c16816106d157600191506106d8565b600 160701b175b6001600160701b03608087901c16836107175780156107125760006106fc82610d1e565b6001955060e203938401607 11901939190911b90505b610721565b600160701b1760721b5b81818161072a57fe5b0490508061074657600160ff1b87871816610 4c35760006104c9565b6001606c1b81101561075457fe5b6000600160731b82101561079457600160721b821015610789576001607 11b821015610781576070610784565b60715b61078c565b60725b60ff1661079d565b61079d82610d1e565b9050836140710181860 
111156107bb57617fff94506000915061084e565b83818601613ffc0110156107d657600094506000915061084e565b83818601613 f8c011015610823578385613ffc011115610801578385613ffc010382901b915061081a565b8385613ffc01101561081a57613ffc8 585030382901c91505b6000945061084e565b6070811115610836576070810382901c91505b6001600160701b03821691508381860 1613f8d010394505b81607086901b888a186001607f1b60801b1660801c6001600160801b0316171760801b955050505050506102d 7565b6000617fff60f084811c8216919084901c8116908214156108cb5780617fff14156108c1576001600160801b0319858116908 51614156103e25784925050506102d7565b84925050506102d7565b80617fff14156108df5783925050506102d7565b6001607f1b6 08086901c90811015906001600160701b031683610905576001935061090c565b600160701b175b6001607f1b608087901c9081101 5906001600160701b0316846109325760019450610939565b600160701b175b82610969576001600160801b03198816600160ff1b1 4610959578761095c565b60005b96505050505050506102d7565b80610989576001600160801b03198916600160ff1b14610959578 861095c565b8486038415158315151415610aac5760708113156109b057899750505050505050506102d7565b60008113156109cf5 7808260008212156109c657fe5b901c9150610a09565b606f198112156109e857889750505050505050506102d7565b60008112156 10a095780600003846000821215610a0157fe5b901c93508596505b92810192600160711b8410610a24576001968701969390931c9 25b86617fff1415610a565784610a3e57617fff60f01b610a48565b6001600160f01b03195b9750505050505050506102d7565b600 160701b841015610a6b5760009650610a78565b6001600160701b03841693505b83607088901b86610a8a576000610a90565b60016 07f1b5b6001600160801b0316171760801b9750505050505050506102d7565b6000811315610ac757600184901b935060018703965 0610ade565b6000811215610ade57600182901b91506001860396505b6070811315610af05760019150610b53565b6001811315610 b185760018103600183036000821215610b0c57fe5b901c6001019150610b53565b606f19811215610b2b5760019350610b53565b6 $00019811215610 b 535760018160000303600185036000821215610 b 4 b 57 f e 5 b 901 c 60010193505 b 818410610 b 64578184039350610$ b6d565b83820393508294505b83610b835750600096506102d795505050505050565b6000610b8e85610d1e565b905080607114156 10bb457600185901c6001600160701b03169450600188019750610c03565b6070811015610bf657607081900380891115610be3578 086901b6001600160701b031695508089039850610bf0565b600098600019019590951b945b50610c03565b6001600160701b03851 694505b87617fff1415610c365785610c1d57617fff60f01b610c27565b6001600160f01b03195b985050505050505050506102d75 65b84607089901b87610c48576000610c4e565b6001607f1b5b6001600160801b0316171760801b985050505050505050506102d75 65b6000617fff60f083901c166140fe811115610c8557600080fd5b613fff811015610c9957600091505061033e565b600160701b6 $001600160701 \mathrm{~b} 03608085901 c 161761406 f 821015610 c c 35761406 f 8290031 c 610 c d 5565 b 61406 f 821115610 c d 55761406 \mathrm{e} 1982011$ b5b6001607f1b608085901c10610d0157600160ff1b811115610cf557600080fd5b600003915061033e9050565b6001600160ff1b0 $3811115610 d 1557600080 f d 5 b 915061033 e 9050565 b 6000808211610 d 2 c 57600080 f d 5 b 6000600160801 b 8310610 d 4157608092831$ c92015b680100000000000000008310610d5957604092831c92015b6401000000008310610d6d57602092831c92015b62010000831 0610d7f57601092831c92015b6101008310610d9057600892831c92015b60108310610da057600492831c92015b60048310610db05 7600292831c92015b600283106102d7576001019291505056fea265627a7a72315820d72739475f1b26be10735eab39ccaf43992cf 00f66b7e7fbdb4a3cad038216a264736f6c63430005100032", \} , "gasUsed": "0xaeb1b"

"subtraces": 0 ,

"traceAddress": [],

"transactionHash": "0x1c98dbb671dbe76b7cb4188d7585296e5adbe0f64fe8144546b4a82081089152",

"transactionPosition": 56,

"type": "create"

\}

] 


\section{Corrected Molecular Dynamics Trajectory Transaction Details}

The details of the transaction and the corresponding blockchain output from the corrected $1 \mathrm{fs}$ molecular dynamics preliminary simulation on the Ethereum blockchain are presented here:

Block Number: 9360161

Hash: 0x13eb9c343f380334262706975c676aa5006ac2103b2132dfba0e1667c7dfddc6

Time Stamp: 01/26/2020 10:26:38 PM Greenwich Mean Time (GMT)

From: 0xC120A82d25325c9700652D6f2288EBD2C4224567

To: 0x9680E167F6221ea0A9dcE066A4f9c769621bC577

Fee: 0.00026 ETH (ca. \$0.04)

Gas Price: 0.0000000011 ETH

Gas Used: 238,619

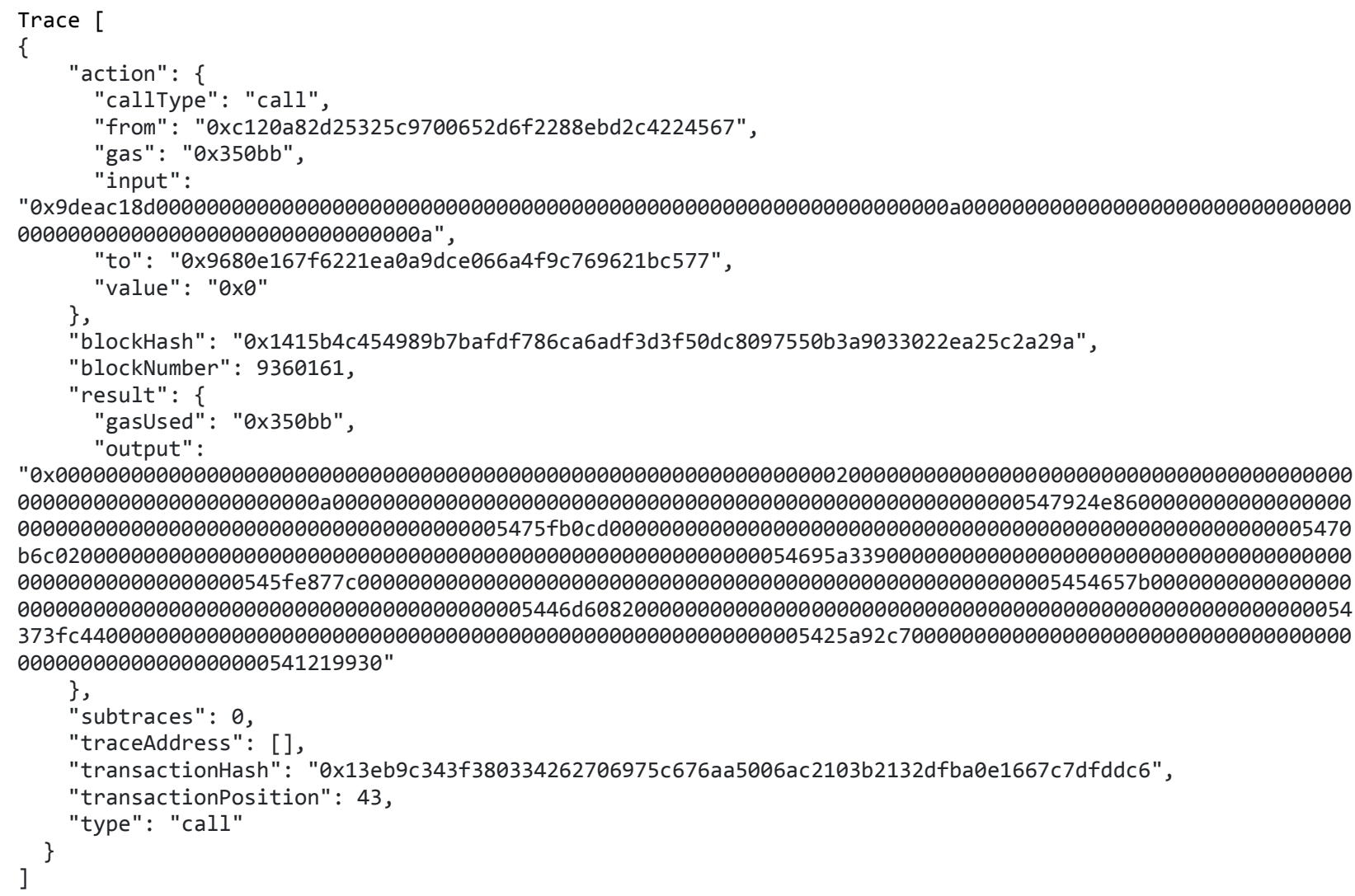


Table S1. Calculated bond lengths $\left(a_{0}\right)$ and simulation times (fs) for the erroneous and corrected molecular dynamics trajectories of 10 steps.

\begin{tabular}{|c|c|c|}
\hline Time & Bond Length (Erroneous) & Bond Length (Corrected) \\
\hline 0.1 & 2.2675607866 & 2.2675607174 \\
\hline 0.2 & 2.2672292763 & 2.2672289997 \\
\hline 0.3 & 2.2666773577 & 2.2666767362 \\
\hline 0.4 & 2.2659059278 & 2.2659048249 \\
\hline 0.5 & 2.2649162402 & 2.2649145212 \\
\hline 0.6 & 2.2637099031 & 2.2637074352 \\
\hline 0.7 & 2.2622888771 & 2.2622855298 \\
\hline 0.8 & 2.2606554714 & 2.2606511172 \\
\hline 0.9 & 2.2588123403 & 2.2588068551 \\
\hline 1.0 & 2.2567624792 & 2.2567557424 \\
\hline
\end{tabular}

Column 1 shows the elapsed simulation time in fs. Columns 2-3 show the calculated carbonoxygen bond lengths (in $\mathrm{a}_{0}$ ) predicted by the erroneous and corrected implementations of the trajectory, respectively. 